\title{
Perfil das instituições de longa permanência para idosos em uma cidade no Estado de São Paulo
}

\section{Profile of long-term care institutions in a city in the State of São Paulo}

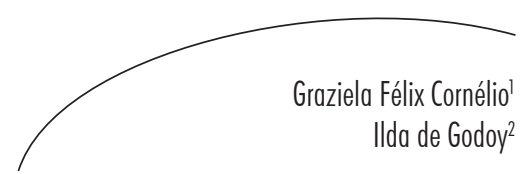

\section{Resumo}

O objetivo deste estudo descritivo, quantitativo e transversal foi caracterizar o perfil de instituições de longa permanência para idosos de Botucatu, SP. Foram entrevistados os responsáveis técnicos e coordenadores de nove instituições existentes no município, com análise de concordância de algumas respostas, e foi aplicada a escala de Katz para avaliar o nível de dependência dos idosos. Os principais resultados foram: deficiência de recursos, principalmente humanos; dificuldades quanto a referência e contrarreferência; falta de rede de apoio social e problemas nas relações com os familiares dos idosos. Além disso, havia restrição de atividades de lazer para os idosos, e tanto a avaliação da qualidade de assistência prestada quanto as atividades de educação continuada para os cuidadores não ocorriam de forma sistematizada. $\mathrm{O}$ estudo mostrou a necessidade de atividades que promovam a autonomia e integração social dos idosos, bem como a capacitação e seleção mais criteriosa dos cuidadores. Portanto, torna-se importante a atuação de profissionais qualificados por meio de uma abordagem interdisciplinar, havendo melhor integração dos serviços com participação de diversos órgãos envolvidos na assistência ao idoso e devem ser mantidos e fortalecidos os vínculos familiares nesse processo.

\section{Abstract}

This descriptive, quantitative and transversal study aimed to characterize the profile of long-term care institutions in the city of Botucatu, State of Sao Paulo, Brazil. We interviewed technical managers and the coordinators of nine institutions in the municipality, with concordance analysis of some answers it was used the Katz instrument to analyze the degree of dependency of elderly. The main results were: lack of resources, especially human; reference and counter-reference difficulties; lack of social support and problems related to their relatives. Moreover, there were leisure activities restrictions for elderly people and both the evaluation of the quality of assistance and continuing education activities that contribute to caregivers did not occur systematically. The study

Palavras-chave: Instituição de Longa Permanência para Idosos. Dependência (Psicologia). Qualidade de Vida. Promoção da Saúde. Recursos Humanos. Educação Continuada.
Key words: Homes for the Aged. Long-term Institution for Elderly. Dependency (Psychology). Quality of Life. Health Promotion. Human Resources. Education, Continuing.

\footnotetext{
Departamento de Enfermagem, Faculdade de Medicina de Botucatu. Universidade Estadual Paulista. Botucatu, SP, Brasil.

2 Programa de Pós-graduação em Enfermagem, Faculdade de Medicina de Botucatu. Universidade Estadual Paulista. Botucatu, SP, Brasil.
} 
showed the need of activities that promote independence and social integration of elderly, and more careful selection and training of caregivers. Therefore, the performance of skilled professionals through an interdisciplinary approach is important, with better integration of services with the participation of several agencies involved in the care of the elderly, and family ties should be maintained and strengthened in this process.

\section{INTRODUÇÃO}

A população brasileira passa por um processo de envelhecimento populacional, e a tendência é que o número de idosos seja equivalente a 32 milhões em 2025, estimando-se uma proporção de $14 \%$ de idosos, com predomínio de doenças crônico-degenerativas. ${ }^{1-4}$ Percebe-se um aumento de idosos com dependência, o que significa que eles estão mais propensos à institucionalização.

Estima-se que, no mundo, cerca de 40\% das pessoas a partir dos 65 anos necessitarão de cuidados em uma instituição de longa permanência para idosos (ILPI) durante sua vida. Projeções realizadas para a população americana mostram que o número de pessoas com necessidade dessa assistência aumentará de 1,5 para 5 milhões no ano de $2030 .^{5,6} \mathrm{Na}$ população brasileira como um todo, foram encontrados 103 mil idosos residentes em ILPIs no ano de 2000, representando aproximadamente $0,8 \%$ da população idosa. ${ }^{7,8}$

A Resolução ANVISA/DC $\mathrm{n}^{\circ}$ 283, de 26/09/2005, aprova o regulamento técnico que define normas de funcionamento para as ILPIs com relação a regulamentação, organização, direção técnica, recursos humanos, processos operacionais, notificação compulsória, monitoramento e avaliação do funcionamento e infraestrutura física destinada a atender os idosos. ${ }^{?}$

Segundo a RDC n ${ }^{\circ}$ 283/05, como atribuições das ILPIs há: desenvolvimento de atividades que estimulem a autonomia, promoção da integração social dos idosos e condições de lazer, tais como atividades físicas, recreativas e culturais. ${ }^{9}$ Além disso, compete às ILPIs desenvolver atividades de educação continuada na área de Gerontologia, para aprimoramento de recursos humanos, bem como realizar avaliação de desempenho e padrão de funcionamento da instituição, com encaminhamento anual, à Vigilância Sanitária, do consolidado de indicadores referentes a agravos à saúde do ano anterior, conforme estabelecido pela resolução. ${ }^{9}$ No entanto, a atenção à saúde do idoso em geral está centrada na cura e na reabilitação, sendo rara em programas sistemáticos de promoção da saúde e de atividades de educação em saúde, com vistas à manutenção da capacidade funcional, principalmente em ILPIs. ${ }^{10}$

Embora o contexto asilar atenda em parte ao idoso em suas necessidades básicas de moradia, alimentação e acompanhamento médico, nem sempre estimula a atividade do idoso. Este tende a se tornar mais introspectivo e isolado do convívio social, convivendo, às vezes, até com pessoas não idosas com doenças incapacitantes, mentais, psiquiátricas e alcoolistas, num ambiente desconfortável. ${ }^{11}$

O objetivo deste estudo, portanto, foi descrever as características das instituições de longa permanência para idosos em Botucatu, SP, para o planejamento de ações que atendam às necessidades dos idosos, visando à melhoria da qualidade de vida.

\section{MÉTODO}

Trata-se de estudo descritivo, quantitativo e transversal, realizado nas nove ILPIs cadastradas pela Vigilância Sanitária de Botucatu, SP, perfazendo um total de 143 idosos institucionalizados. Foram incluídas todas as instituições existentes no município, classificadas como instituições de longa permanência para idosos, conforme os critérios estabelecidos para esta denominação, obtidos por meio de informações da Vigilância Sanitária do município de Botucatu. No momento da pesquisa, havia 143 idosos institucionalizados, sendo $71 \%$ do sexo feminino. A coleta de dados foi realizada no período de novembro de 2009 a março de 2010. 
Foram entrevistados nove responsáveis técnicos e oito coordenadores; salienta-se que, em uma instituição não havia coordenador. Considerou-se como coordenador aquele que acompanhava mais diretamente os cuidados ao idoso e coordenava os demais membros da equipe, podendo ou não ser o proprietário da instituição. Utilizou-se como instrumento de coleta de dados um questionário elaborado com questões fechadas e abertas, que foram categorizadas de acordo com a similaridade das respostas.

Considerando as atribuições das ILPIs de desenvolver atividades que estimulem a autonomia, bem como promover a integração social e as condições de lazer, utilizou-se a escala ou índice de Katz ${ }^{12,13}$ para a classificação do grau de dependência dos idosos abrigados.

Em se tratando de atividades de lazer, optouse pela classificação segundo Dumazedier ${ }^{14} \mathrm{e}$ Marcellino, ${ }^{15}$ por se tratar de referenciais teóricos clássicos para a classificação de atividades de lazer e utilizados atualmente por vários autores. ${ }^{16-18}$ Portanto, as respostas foram agrupadas em seis categorias de atividades: artístico (ir ao cinema, teatro, acesso à literatura); intelectual (busca de conhecimentos, científicos ou não como jornais, revistas); manual (lavar o carro nos finais de semana, cultivar hortaliças, crochê, tricô); físico (caminhadas, ginástica, esporte e atividades correlatas, executadas de maneira formal ou informal, em espaços tecnicamente planejados, como pistas, academias); social (convívio doméstico, com jogos e passeios com filhos, visitas a parentes e amigos, movimentos culturais) e turístico (viagens, passeios). ${ }^{14,15}$

Utilizou-se o programa SAS Windows versão 9.1 para o cálculo das frequências e a análise de concordância, com base no coeficiente Kappa ${ }^{19,20}$ das respostas entre os coordenadores e responsáveis da mesma instituição, sendo que valores maiores de 0,75 representam excelente concordância e abaixo de 0,40 , baixa concordância.

Este estudo foi aprovado pelo Comitê de Ética e Pesquisa da Faculdade de Medicina de Botucatu, protocolo CEP no 3.345/2009, e todos os participantes foram devidamente esclarecidos quanto aos objetivos da pesquisa, sendo entrevistados somente após a assinatura do Termo de Consentimento Livre e Esclarecido.

\section{RESULTADOS}

Dos entrevistados, dois dos responsáveis técnicos atuavam em mais de uma instituição e um coordenador, em duas instituições. Os dados, no entanto, foram considerados individualmente, como observações por instituiç̧ão.

Dos responsáveis, quatro eram enfermeiros, três médicos e dois assistentes sociais. Quanto aos coordenadores, um era enfermeiro, quatro eram técnicos de enfermagem, dois tinham curso superior incompleto e um-tinha ensino fundamental incompleto.

\section{Características das instituições quanto a natureza jurídica, profissionais e usuários}

Das nove ILPIs, apenas uma foi reconhecida como filantrópica e as demais como de caráter privado. Com relação aos profissionais de saúde com nível superior, em todas as instituições foi referida a existência de enfermeiro. $\mathrm{Na}$ instituição filantrópica, existiam outros profissionais como assistente social, também presente em mais quatro instituições; médico e fisioterapeuta, em mais duas instituições; psicólogo e terapeuta ocupacional, ausente nas demais.

O número total de cuidadores era 43 , variando de dois a dez por instituição, sendo que somente $9,3 \%$ deles realizaram curso de cuidador de idoso. Os responsáveis e coordenadores foram questionados quanto ao critério de seleção desses cuidadores. Os critérios mais adotados pelos coordenadores foram entrevista (100\%), seguida pela avaliação do currículo (87,5\%) e experiência anterior (62,5\%). Por outro lado, quando os responsáveis foram abordados, outros critérios foram destacados, como indicação $(92,5 \%)$, seguida por entrevista $(50 \%)$ e experiência anterior (50\%). Quando abordados quanto ao critério de curso na área da saúde, os entrevistados de três instituições, incluindo a 
filantrópica, consideravam a formação na área da enfermagem como pré-requisito importante. Destaca-se que em quatro instituições, não envolvendo a filantrópica, os cuidadores desempenhavam também funções relacionadas ao preparo do alimento e atividades de limpeza.

Com relação à capacitação dos cuidadores, a maior parte dos entrevistados, 75\% dos coordenadores e 55,6\% dos responsáveis afirmaram que há treinamentos nas instituições, com frequência esporádica, sendo os temas mais abordados: higiene, curativo, primeiros-socorros e nutrição. Os profissionais que relataram não realizar treinamentos apontaram como motivos: falta de necessidade, por $50 \%$ dos coordenadores e $25 \%$ dos responsáveis; inabilidade para ensinar, para 50\% dos coordenadores; falta de recursos financeiros, para 50\% dos responsáveis; ou problemas relacionados a recursos humanos, para 25\% dos responsáveis. Sobre cursos realizados externamente, $62,5 \%$ dos coordenadores referiram que procuram informar os cuidadores, estimulando a participação; 44,5\% dos responsáveis e 37,5\% dos coordenadores destacaram que não ficam sabendo de muitos cursos, que passam despercebidamente.

Havia 143 idosos institucionalizados no momento da pesquisa, sendo $71 \%$ do sexo feminino e quatro instituições abrigavam cinco pessoas na faixa etária menor de 60 anos. A distribuição do grau de dependência dos idosos segundo escala de Katz ${ }^{12,13}$ está apresentada na tabela 1. Nota-se que a única instituição em que não ocorreu predomínio de dependentes totais foi a instituição A (filantrópica).

Tabela 1 - Distribuição do número de idosos segundo o grau de dependência. Botucatu-SP, 2010.

\begin{tabular}{|c|c|c|c|c|c|c|}
\hline \multirow{2}{*}{$\begin{array}{c}\text { Grau de dependência } \\
\text { Instituição }\end{array}$} & \multicolumn{2}{|c|}{ Dependência total } & \multicolumn{2}{|c|}{ Dependência parcial } & \multicolumn{2}{|c|}{ Independente } \\
\hline & $\mathrm{n}$ & $\%$ & $\mathrm{n}$ & $\%$ & $\mathrm{n}$ & $\%$ \\
\hline $\mathrm{A}$ & 0 & 0,0 & 35 & 55,6 & 28 & 44,4 \\
\hline B & 4 & 57,1 & 2 & 28,6 & 1 & 14,3 \\
\hline $\mathrm{C}$ & 16 & 66,7 & 0 & 0,0 & 8 & 33,3 \\
\hline $\mathrm{D}$ & 6 & 60,0 & 1 & 10,0 & 3 & 30,0 \\
\hline $\mathrm{E}$ & 5 & 62,5 & 2 & 25,0 & 1 & 12,5 \\
\hline $\mathrm{F}$ & 5 & 83,3 & 1 & 16,7 & 0 & 0,0 \\
\hline G & 5 & 71,4 & 1 & 14,3 & 1 & 14,3 \\
\hline $\mathrm{H}$ & 7 & 77,8 & 2 & 22,2 & 0 & 0,0 \\
\hline I & 6 & 66,7 & 3 & 33,3 & 0 & 0,0 \\
\hline
\end{tabular}

\section{Assistência ao idoso}

Quanto à realização de atividades de lazer com os idosos nas instituições, a maioria dos coordenadores $(62,5 \%)$ afirmou a ausência; entretanto, a maioria dos responsáveis $(55,6 \%)$ afirmou a presença, com periodicidade semanal ou mensal. Dentre os tipos, destacou-se a atividade social, referida por $100 \%$ dos coordenadores, que responderam afirmativamente quanto ao desenvolvimento de atividades de lazer e $60 \%$ dos responsáveis, tais como: aniversários, grupos de convivência, dinâmica recreativa, passeios com filhos, visitas, brincadeiras e jogos. As outras atividades promovidas com menor frequência foram artísticas (filmes, música); intelectuais (leitura, palavras cruzadas); manuais (oficinas de artesanato, crochê); foi relatada por uma instituição a realização de atividade turística (passeios a outras cidades). Ressalta-se que a atividade física foi ausente em todas as instituições. 
A existência de dificuldades e necessidades quanto ao gerenciamento, principalmente de recursos humanos, foi mencionada por $87,5 \%$ dos coordenadores e 100\% dos responsáveis. Houve predomínio de relato referente ao número insuficiente de profissionais e à falta de capacitação, com necessidade de cursos ou espaços para trocas de experiências entre as ILPIs. As dificuldades referentes a recursos materiais foram relatadas por $77,8 \%$ dos responsáveis, como a deficiência de equipamentos gerais ou a falta de rede de apoio. Foram destacadas, por $66,67 \%$ dos responsáveis, dificuldades quanto ao usuário, tais como ausência ou interferência do familiar do idoso.

Quando os responsáveis foram abordados com relação às dificuldades no cuidado do idoso, somente um referiu ausência, enquanto a maioria $(75 \%)$ referiu dificuldades quanto à referência e contrarreferência ou rede de apoio. Para os coordenadores, a principal dificuldade citada $(50 \%)$ foi quanto às condições de trabalho limitadas por déficit financeiro, com necessidade de mais funcionários, material e/ou estrutura física adequada.
Dentre as facilidades, $62,5 \%$ dos coordenadores referiram o preparo da equipe em termos de competência, experiência, aptidão, colaboração ou humanização no atendimento e $66,7 \%$ dos responsáveis destacaram a aceitação dos idosos com reconhecimento e valorização do cuidado prestado a eles.

Quanto à avaliação da qualidade da assistência ao idoso, um coordenador e um responsável técnico referiram ausência de avaliação. $\mathrm{O}$ modo de avaliação limitou-se, pela maior parte $(85,7 \%$ dos coordenadores e $50 \%$ dos responsáveis), a observação ou verbalização entre a equipe, com inexistência de instrumento.

Por último, realizou-se a análise de concordância, com base no coeficiente de $\mathrm{Kappa}^{19,20}$ das respostas entre coordenadores e responsáveis da mesma instituição das questões relacionadas à avaliação da qualidade da assistência e a capacitação. Os valores dos coeficientes foram predominantemente abaixo de 0,40 , representando baixa concordância, de acordo com os resultados apresentados na tabela 2 .

Tabela 2 - Coeficiente de Concordância Kappa para coordenadores e responsáveis quanto a variáveis relacionadas à capacitação e avaliação da qualidade da assistência. Botucatu-SP, 2010.

\begin{tabular}{lcc}
\hline \multicolumn{1}{c}{ Variáveis } & Kappa & IC $95 \%$ \\
\hline Capacitação & & \\
Realização de treinamentos & $-0,197$ & $(-0,64 ; 0,25)$ \\
Temas & & \\
Curativo & $-0,428$ & $(-0,95 ; 0,09)$ \\
Nutrição & 0,400 & $(-0,15 ; 0,96)$ \\
Legislação & $-0,191$ & $(-0,52 ; 0,14)$ \\
Locomoção & $-0,250$ & $(-0,83 ; 0,33)$ \\
Higiene & 0,138 & $(-0,13 ; 0,41)$ \\
Psico & $-0,139$ & $(-0,39 ; 0,11)$ \\
Socorros & $-0,400$ & $(-0,97 ; 0,16)$ \\
Outros & 0,091 & $(-0,47 ; 0,65)$ \\
Avaliação da qualidade da assistência & & \\
Presença de avaliação & 0,014 & $(-0,31 ; 0,34)$ \\
Existência de instrumento & 0,348 & $(-0,08 ; 0,78)$ \\
\hline
\end{tabular}




\section{DISCUSSÃO}

Segundo a RDC no $283 / 05$, ${ }^{\circ}$ o responsável técnico deve possuir formação de nível superior, exigência contemplada em todas as instituições deste estudo. Além disso, as ILPIs devem ter em seu quadro funcionários com vínculo formal para limpeza, serviços de alimentação e de lavanderia, de acordo com a área e o número de idosos da instituição.

Neste estudo, havia quatro instituições com deficiência desses profissionais, o que pode comprometer a qualidade da assistência. Estes dados são semelhantes aos resultados do estudo de Ribeiro et al. ${ }^{21}$ realizado em instituições filantrópicas, onde foi encontrada duplicidade de funções, como fazer a limpeza da instituição e lavar a roupa dos idosos, mostrando, por meio dos relatos de alguns profissionais, a interferência da falta de divisão de funções entre cuidadores e outros profissionais na qualidade do cuidado, devido à falta de tempo.

Destaca-se também a falta de cuidadores com formação na área da saúde, incluindo o curso cuidador de idoso, apesar de esse curso não ser considerado pré-requisito no processo de seleção na maioria das instituições. Essas deficiências não foram relatadas na instituição filantrópica. A deficiência de cuidadores com cursos específicos para prestar cuidados aos idosos e a não exigência deste critério para a contratação na cidade de Botucatu podem ser justificadas pelo não oferecimento de cursos com regularidade e pela baixa adesão do público-alvo.

Em se tratando de atividades de lazer, estudos mostram que poucos idosos praticam regularmente atividade física, com predominância da caminhada. ${ }^{22,23}$ Mesmo esta se destacando por ser uma atividade física natural que ocorre sem custos, nas instituições onde foi realizado este estudo não havia promoção de atividade física, justificada pela necessidade de espaços planejados para recreação e considerações quanto a debilidade física ou resistência de idosos institucionalizados.
Há necessidade de políticas públicas para viabilizar e ampliar instrumentos de lazer de uso coletivo, para sensibilizar as pessoas a participarem de atividades planejadas, assim como promover políticas de inclusão social. Para isso, programas de promoção de atividades físicas para a população idosa devem considerar as variáveis relacionadas às estruturas públicas e privadas (áreas verdes, centros de lazer, parques, academias, praças, quadras, postos de saúde e bancos), locais que congregam reuniões sociais (igrejas), suporte social (ser convidado por amigos para praticar atividade física) e percepção de segurança. ${ }^{22,24}$

Em uma ILPI localizada na cidade de Rio Grande, RS, a maioria dos idosos não realizava nenhuma atividade física. Atividades como artesanato, bingo, dança, passeio, leitura, grupo religioso, costura, assistir televisão, ouvir rádio e leituras eram esporádicas ou ainda praticadas individualmente, pois não havia grupos ou atividades de lazer sistemáticas na instituição. Dessa forma, dentre os diagnósticos de enfermagem (NANDA), identificouse nesta pesquisa: "déficit nas atividades de lazer relacionado à dificuldade de acesso ou participação nas atividades de lazer evidenciado por verbalização de tédio e desejo de que tivesse alguma coisa para fazer". ${ }^{25}$

Outra pesquisa realizada com idosos institucionalizados de duas ILPIs concluiu que programas de estimulação a partir da prática de atividades cognitivas e atividades físicas produzem efeitos positivos na capacidade cognitiva geral e na depressão de idosos institucionalizados, sugerindo a necessidade de mais estudos na área. ${ }^{26} \mathrm{O}$ lazer é um dos meios de socialização, de saúde e de qualidade de vida dos idosos. ${ }^{18}$ Dessa forma, entende-se a importância da introdução ou expansão de atividades de lazer de forma regular em todas as instituições estudadas.

Nas instituições asilares de longa permanência, a dependência física é muitas vezes estimulada, pois os próprios funcionários 
preferem ajudar os idosos em suas atividades, quando esses já apresentam inabilidade para executar tarefas simples, embora não sejam incapazes para fazê-las. ${ }^{27}$ Tais fatores podem explicaropredomínio deidosos com dependência total, exceto na instituição filantrópica, onde atuavam também profissionais como psicólogo e terapeuta ocupacional, inexistentes nas demais instituições.

Atitudes paternalistas e assistencialistas podem gerar efeitos negativos para a autonomia dos idosos, pois desencadeiam a dependência do cuidado profissional. ${ }^{28}$ De acordo com a Política Nacional de Saúde da Pessoa Idosa:

A prática de cuidados às pessoas idosas exige abordagem global, interdisciplinar e multidimensional, que leve em conta a grande interação entre os fatores físicos, psicológicos e sociais que influenciam a saúde dos idosos e a importância do ambiente no qual está inserido... As intervenções devem ser feitas e orientadas com vistas à promoção da autonomia e independência da pessoa idosa, estimulando-a para o autocuidado. ${ }^{29}$

Para isso, é imprescindível preparar/ capacitar os profissionais da saúde, uma vez que estão diretamente envolvidos no cuidado. Tal capacitação implica despertar no profissional da saúde o reconhecimento do idoso cidadão, ou seja, um profissional conhecedor da realidade social e de saúde desse estrato populacional, das tecnologias existentes, dos recursos disponíveis e dos dispositivos legais como instrumentos factíveis para o desenvolvimento de ações de saúde. O profissional deve estar preparado para reconhecer no idoso a potencialidade para o autocuidado, a necessidade de interdependência para o cuidado e a importância de preservar a autonomia. ${ }^{28}$

No que diz respeito ao gerenciamento de recursos, as principais dificuldades foram referentes a recursos humanos, especialmente relacionados ao número insuficiente. Neste estudo, a maioria das ILPIs privadas (segundo $87,5 \%$ dos coordenadores e $88,9 \%$ dos responsáveis técnicos) depende de recursos próprios com ou sem a participação do familiar. Segundo Camarano \& Kanso, ${ }^{30}$ a maior parcela das despesas das ILPIs é destinada ao pagamento de seus funcionários, o que corresponde a 52,5\% do total. Dessa forma, o custo elevado justifica a deficiência de profissionais.

De acordo com levantamento feito por Camarano \& Kanso, ${ }^{30}$ das ILPIs brasileiras criadas entre 2000 e 2009, a maioria é privada com fins lucrativos (57,8\%) e vivem principalmente do recurso aportado pelos residentes e/ou familiares. No presente estudo, somente duas instituições recebiam doações e dois coordenadores (25\%) referiram utilizar recursos públicos, sendo um pertencente à filantrópica e o outro a uma de caráter privado que utiliza tal recurso somente para aquisição de medicamentos.

Dificuldades quanto ao cuidado do idoso também foram citadas pelos coordenadores e responsáveis, exceto em uma instituição. As dificuldades apontadas pelos coordenadores foram mais direcionadas a fatores internos, como condições de trabalho caracterizadas pela falta de recursos, enquanto que, para os responsáveis, estavam ligadas a fatores externos relacionados a referência e contrarreferência de idosos (encaminhamento/direcionamento para outros serviços, em caso de internações ou consultas e retornos) ou rede de apoio (suporte familiar e social) com deficiência de recursos financeiros e necessidade de recursos públicos.

De acordo com a RDC no 283/05, ${ }^{\circ}$ ILPIs devem elaborar, a cada dois anos, um Plano de Atenção Integral à Saúde dos Residentes, em articulação com o gestor local de saúde, indicando os recursos de saúde disponíveis para cada residente, em todos os níveis de atenção, públicos ou privados, bem como referências, caso se faça necessário.

Para Nardi \& Oliveira, ${ }^{31}$ faz-se necessário formar redes de apoio, integrando sistemas informais e formais, sugerindo treinamento das equipes da Estratégia de Saúde da Família (ESF), com foco de atenção ao idoso e seu cuidador, 
e ainda a articulação com outras instituições locais. No estudo de Creutzberg et al., ${ }^{10}$ realizado em ILPIs de caráter público ou privado e sem fins lucrativos, foram percebidas dificuldades nas relações entre as ILPIs e o Sistema Único de Saúde (SUS), como falta de humanização no atendimento e dificuldade de acesso a recursos materiais disponíveis. Além disso, em casos de atendimento em emergências ou hospitalização, houve desrespeito e não priorização do idoso, assim como a problemática da decisão de hospitalizá-lo e falta de envolvimento familiar nesse processo, desconsiderando-se o possível direito de autonomia do idoso. ${ }^{10}$

A clareza acerca dos serviços, dos processos de referência e contrarreferência, das possibilidades e a estimulação por meio da proposição de alternativas pode resultar em acoplamento estrutural mais bem-sucedido entre as ILPIs e o SUS. ${ }^{10}$ Percebe-se, contudo, que é necessário estabelecer responsabilidades dos dois segmentos voltados à atenção ao idoso, principalmente em se tratando de ILPIs privadas, com melhor integração destes serviços, de forma a garantir a continuidade do tratamento com integralidade das ações, destacando também o papel de promoção à saúde.

Dificuldades quanto ao familiar também foram mencionadas por coordenadores e responsáveis na mesma proporção, como atitudes de ausência ou cobrança. Creutzberg et $a{ }^{32}$ explicam que a dificuldade de relação entre as ILPIs e os familiares de idosos ocorre devido ao processo de institucionalização mal trabalhado pelas ILPIs, de um lado, e mal elaborado pela família, de outro. Segundo os autores ${ }^{32}$ as famílias não se envolvem no auxílio funcional pelo fato de depositarem na instituição a tarefa de cuidar, mas há também limites nas ILPIs à participação delas, como a restrição de horário para visitas familiares. A família, junto com seu idoso, deve ser o foco da instituição, sendo considerada também como usuária. Devem ser buscadas alternativas para a inclusão desse importante recurso, com consequente repercussão nas ILPIs, como a qualificação do cuidado ao idoso. ${ }^{32}$
Quanto às facilidades no cuidado ao idoso, a aceitação dos idosos demonstrada por manifestações de reconhecimento e valorização do atendimento prestado a eles leva a refletir sobre a passividade do idoso no processo de cuidado, levando em conta o grau de dependência predominante com base na aplicação da escala de Katz. Além disso, muitos coordenadores consideram que a equipe está preparada para cuidar do idoso com competência, humanização da assistência, mas, por outro lado, muitos referiram necessidades referentes à capacitação de profissionais.

Embora a maioria dos responsáveis e coordenadores referisse a realização de treinamentos internos, houve profissionais de algumas instituições que referiram ausência por motivos referentes à falta de estrutura das instituições ou por julgá-los desnecessários. Além disso, a realização de atividades de educação continuada de forma sistematizada se torna duvidosa devido à baixa concordância, na análise do coeficiente de Kappa ${ }^{19,20}$ das respostas obtidas entre os coordenadores e responsáveis da mesma instituição, exceto sobre nutrição, que obteve concordância mediana. Essa baixa concordância pode sugerir a falta de integração entre os profissionais, com distanciamento do processo de trabalho.

No Brasil, a avaliação da qualidade dos cuidados oferecidos nas ILPIs brasileiras não é feita de forma sistemática, e aponta resultados conflitantes. Em função desta dificuldade de avaliação, alguns autores determinaram a qualidade da atenção prestada aos idosos com base na estrutura das mesmas referente à área física, aos recursos humanos e às normas técnico-administrativas. ${ }^{33}$ Neste estudo, a baixa concordância na aplicação do coeficiente de concordância Kappa ${ }^{19,20}$ pode sugerir que a avaliação da qualidade da assistência ao idoso nas ILPIs também não ocorre de forma sistemática.-

Como limitação desta pesquisa, ressalta-se o fato de haver, entre os entrevistados, responsáveis e coordenadores que atuavam em mais de uma instituição concomitantemente. 


\section{CONSIDERAÇÕES FINAIS}

Este estudo evidenciou a falta de definição de critérios de seleção para a contratação de cuidadores e problemas de estrutura nas ILPIs, em termos de recursos físicos, humanos, materiais e financeiros, com destaque para os aspectos quantitativos e qualitativos.

Com os resultados encontrados, é possível inferir que não existe avaliação da qualidade

\section{REFERÊNCIAS}

1. Paschoal SMP, Franco RP, Salles RFN. Epidemiologia do envelhecimento. In: Papaléo Netto M. Tratado de Gerontologia. 2. ed. rev. ampl. São Paulo: Atheneu; 2007. p. 39-56.

2. Garcia MAA, Rodrigues MG, Borega RS. O envelhecimento e a saúde. Rev Ciênc Med 2002;11(3):221-31.

3. Chaimowicz F. A saúde dos idosos brasileiros às vésperas do século XXI: problemas, projeções e alternativas. Rev Saúde Pública 1997;31(2):184-200.

4. Papaléo Netto M. Processo de envelhecimento e longevidade. In: Papalélo Netto M. Tratado de Gerontologia. 2. ed. rev. ampl. São Paulo: Atheneu; 2007. p. 3-14.

5. Kemper P, Murtaugh CM. Lifetime use of nursing home care. N Engl J Med 1991;324:595-600.

6. Villas Bôas PJF, Ferreira ALA. Infecção em idosos internados em instituição de longa permanência. Rev Assoc Med Bras 2007;53(2):126-9.

7. Camarano AA, coordenadora. Características das Instituições de Longa Permanência para Idosos: região Norte. Brasília: IPEA; 2007. 99 p.

8. Christophe M. Instituições de longa permanência para idosos no Brasil: uma opção de cuidados de longa duração? [Dissertação de Mestrado]. Rio de Janeiro: Escola Nacional de Ciências Estatísticas, Programa de Pós-Graduação em Estudos Populacionais e Pesquisas Sociais; 2009.

9. Resolução da Diretoria Colegiada. RDC/ANVISA n. 283, de 26 de setembro de 2005. 2005 [acesso em 15 maio 2009]. Disponível em: htt;p//www.portal.saude. al.gov.br/suvisa/files/rdc_283.pdf

10. Creutzberg M, Gonçalves LHT, Sobottka EA, Ojeda BS. A instituição de longa permanência para de assistência prestada, nem a realização de atividades de educação continuada de forma sistematizada nas ILPIs. Assim, tornase importante a atuação de profissionais qualificados, por meio de uma abordagem interdisciplinar e melhor integração dos serviços, com participação de diversos órgãos envolvidos na assistência ao idoso. Nesse processo, devem ser mantidos e fortalecidos os vínculos familiares. idosos e o sistema de saúde. Rev Latino-Am Enferm 2007;15(6):1144-9.

11. Pestana LC, Santo FHE. As engrenagens da saúde na terceira idade: um estudo com idosos asilados. Rev Esc Enferm USP 2008;42(2):268-75.

12. Carvalho GA, Peixoto NM, Capella PD. Análise comparativa da avaliação funcional do paciente geriátrico institucionalizado por meio dos protocolos de Katz e Tinetti. EFDeportes.com Rev Digital [periódico na Internet]. 2007 [acesso em 2 jul 2010];12(114):[aproximadamente 4 p.]. Disponível em: http://www.efdeportes.com/ efd114/avaliacao-funcional-do-paciente-geriatricoinstitucionalizado.htm

13. Duarte YAO, Andrade CL, Lebrão ML. O Índex de Katz na avaliação da funcionalidade dos idosos. Rev Esc Enferm USP 2007;41(2):317-25.

14. Dumazedier J. Valores e conteúdos culturais do lazer. São Paulo: SESC; 1980.

15. Marcellino NC. Lazer e humanização. Campinas: Papirus; 1995.

16. Lunardi R, Silva AP. Planejamento público urbano do lazer para idosos em Santa Maria - RS. Disciplinarum Scientia 2003;4(1):141-56.

17. Paim MCC, Silva CN, Jardim EJS, Tonetto G. Atividades de lazer praticadas por acadêmicos da UFSM no seu tempo livre. EFDeportes.com Rev Digital [periódico na Internet]. 2004 [acesso em 20 maio 2013];10(69):[aproximadamente 3 p.]. Disponível em: http://www.efdeportes.com/efd69/ufsm.htm

18. Brandão JS. Lazer para o idoso ativo como fator de qualidade de vida no processo de envelhecimento [tese de Doutorado]. Porto Alegre: Programa de PósGraduação em Gerontologia Biomédica da Pontifícia Universidade Católica; 2009. 
19. Kotz S, Johnson NL. Encyclopedia of statistical sciences, v. 4. New York: John Wiley \& Sons; 1983. p. $352-4$.

20. Landis JR, Koch GG. The measurement of observer agreement for categorical data. Biometrics 1977;33(1):159-74.

21. Ribeiro MTF, Ferreira RC, Magalhães CS, Moreira AN, Ferreira EF. Processo de cuidar nas instituições de longa permanência: visão dos cuidadores formais de idosos. Rev Bras Enferm 2009;62(6):870-5.

22. Almeida MMG, Moreira RF, Araújo TM, Pinho PS. Atividades de lazer entre idosos, Feira de Santana, Bahia. Rev Baiana Saúde Pública 2005;29(2):339-52.

23. Hughes JP, McDowell MA, Brody DJ. Leisure-time physical activity among US adults 60 or more years of age: results from NHANES 1999-2004. J Phys Act Health 2008;5(3):347-58.

24. Salvador EP, Florindo AA, Reis RS, Costa EF. Percepção do ambiente e prática de atividade física no lazer entre idosos. Rev Saúde Pública 2009;43(6):972-80.

25. Santos SSC, Feliciani AM, Silva BT. Perfil de idosos residentes em instituição de longa permanência: proposta de ações de enfermagem/saúde. Rev RENE 2007;8(3):26-33.

26. Tavares L, Takase E, Chaves AA, Schmidt B, Guidoni BC. Programas de estimulação em idosos institucionalizados: efeitos da prática de atividades cognitivas e atividades físicas. EFDeportes.com Rev Digital [periódico na Internet]. 2009 [acesso em 20 jun 2010];13(129):[aproximadamente 5 p.]. Disponível em: http://www.efdeportes.com/efd129/programasde-estimulacao-em-idosos-institucionalizados.htm
27. Pavarini SCI. Dependência comportamental na velhice: uma análise do cuidado prestado ao idoso institucionalizado [tese de Doutorado]. Campinas: Faculdade de Educação da Universidade Estadual de Campinas; 1996.

28. Martins JJ, Schier J, Erdmann A L, Albuquerque G L. Políticas públicas de atenção à saúde do idoso: reflexão acerca da capacitação dos profissionais da saúde para o cuidado com o idoso. Rev Bras Geriatr Gerontol [Internet]. 2007 [acesso em 22 maio 2013];10(3):371-82. Disponível em: http:// revista.unati.uerj.br/scielo.php?script $=$ sci_ arttext\&pid=S1809-98232007000300009\&lng=pt.

29. Brasil. Ministério da Saúde. Portaria n. 2.528, de 19 de outubro de 2006. Aprova a Política Nacional de Saúde da Pessoa Idosa. Brasília: MS; 2006.

30. Camarano AA, Kanso S. As instituições de longa permanência para idosos no Brasil. Rev Bras Estud Popul 2010;27(1):232-5

31. Nardi EFR, Oliveira MLF. Conhecendo o apoio social ao cuidador familiar do idoso dependente. Rev Gaúcha Enferm 2008;29(1):47-53.

32. Creutzberg M, Gonçalves LHT, Sobottka EA, Santos BRL. A comunicação entre a família e a Instituição de Longa Permanência para Idosos. Rev Bras Geriatr Gerontol 2007;10(2):147-60.

33. Born T, Boechat NS. A qualidade dos cuidados ao idoso institucionalizado. In: Freitas EV, Py L, Cançado FAX, Doll J, Gorzoni ML, organizadores. Tratado de Geriatria e Gerontologia. Rio de Janeiro: Guanabara koogan; 2006. p. 1131-41. 